Somerville et al report an interesting systematic review of usual care in low back pain primary care trials. ${ }^{1}$ They highlight the variability that currently exists in the usual care packages, which function as the comparator treatment in many trials, and point out that the content of treatment in this arm is often inadequately described. They draw our attention to the difficulties associated with the interpretation of results when this comparator treatment is poorly described. We hope their message does not fall on deaf ears.

Notwithstanding this, we feel that their interpretation that the speed at which the Roland Morris Disability Questionnaire (RMDQ) score changes over time is dependent on the duration of low back pain, may be inaccurate. The clinical trajectories in several acute/sub-acute trial arms to trajectories from two small chronic low back pain trials were compared and Somerville et al note a more rapidly improving trajectory in the acute/sub-acute arms. ${ }^{2,3}$ Meng et al's report ${ }^{2}$ on a trial of acupuncture $(n=24)$ for chronic low back pain in older patients describes follow-up results at 2, 6, and 9 weeks; rather than the 30 week follow-up period indicated by Somerville et al.

Licciardone's report of a trial of osteopathic manipulation $(n=20)^{3}$ did not provide point estimates for follow-up RMDQ scores in their usual care group; rather, it was stated 'there was no significant decrease'. This was represented as Figure 1 in Somerville et al's report as a stable trajectory. ${ }^{1}$

We have done a new analysis of data from the UK BEAM trial ${ }^{4}$ (Brealey et $a^{5}{ }^{5}$ in the original paper), extracting data on outcomes for those in the usual care group of this trial with pain lasting for more than 3 months $(n=205)$.

Furthermore, we obtained data from the usual care arm of Licciardone et al's trial of osteopathic manipulation (thanks to John Licciardone). For completeness, we present a figure including these data (Figure 1).

The usual care arm in the UK BEAM trial was nearly eight times the size of the

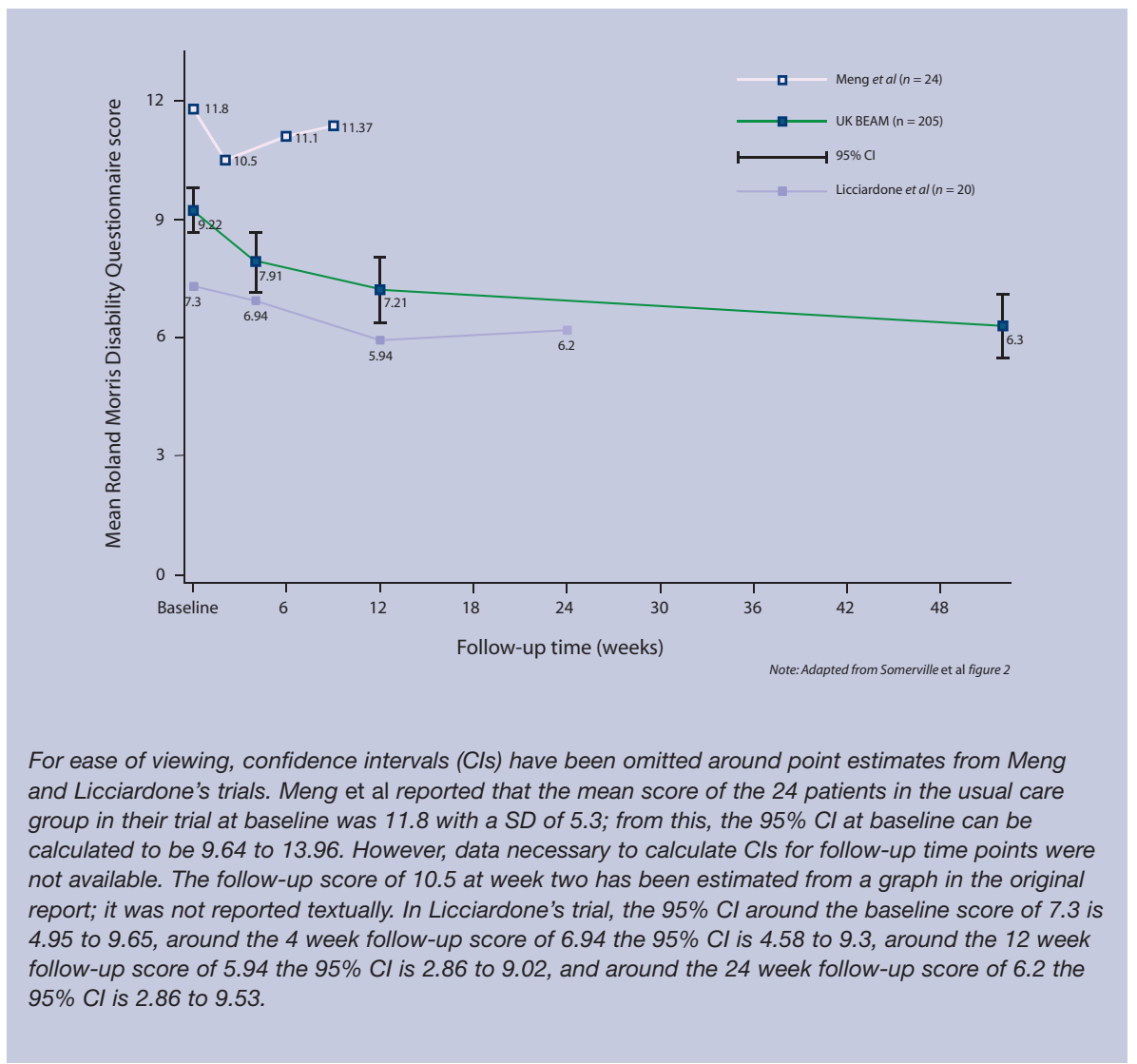

two previously included studies combined. Data from patients with chronic pain show a markedly different trajectory from those described in Somerville et al's report (Figures 1 and 2). It more closely mimics the acute/subacute trajectories they presented. In contrast to their conclusion, we conclude that patients in usual care arms are likely to improve in trials of both acute and chronic back pain.

\section{Robert Froud,}

Barts \& The London School of Medicine and Dentistry, Queen Mary University of London, Institute of Health Sciences Education, London E1 2AD. E-mail: r.j.froud@qmul.ac.uk

\section{Martin Underwood,}

Warwick Clinical Trials Unit, Warwick Medical School, Gibbet Hill Road, Coventry CV4 7AL.

\section{REFERENCES}

1. Somerville S, Hay E, Lewis M, et al. Content and outcome of usual primary care for back pain: a systematic review. Br J Gen Pract 2008; 58(556): 790-797, i-vi.

2. Meng CF, Wang D, Ngeow J, et al. Acupuncture for chronic low back pain in older patients: a randomized, controlled trial. Rheumatology (Oxford) 2003; 42(12): 1508-1517.

3. Licciardone JC, Stoll ST, Fulda KG, et al. Osteopathic manipulative treatment for chronic low back pain: a randomized controlled trial. Spine 2003; 28(13): 1355-1362.

4. UK BEAM Trial Team. United Kingdom back pain exercise and manipulation (UK BEAM) randomised trial: effectiveness of physical treatments for back pain in primary care. BMJ 2004; 329(7479): 1377.

5. Brealey S, Burton K, Coulton S, et al. UK Back pain Exercise and Manipulation (UK BEAM) trial national randomised trial of physical treatments for back pain in primary care: objectives, design and interventions. BMC Health Services Research 2003; 3(1): 16 .

DOI: 10.3399/bjgp09X419574

\section{Author's response}

I welcome the comments and additional data provided by Froud and Underwood. I quite agree with them that the endpoint for data collection in Meng et al's report' was at 9 weeks rather than 30 weeks as represented in the published version of in Figure $2 b$ of our paper. ${ }^{2}$ The version of our paper that was accepted for 
publication in fact shows the endpoint correctly at 9 weeks. The error appears to have occurred during the production phase at the journal and was not spotted by me in the final proof. I apologise and take full responsibility for this. However, this does not alter the fact that the Roland Morris Disability Questionnaire (RMDQ) scores in Meng et al's study of people with chronic back pain did not improve significantly during the course of their study.

For Froud and Underwood to obtain the data that they present in Figure 1 overleaf they have had to re-interpret results from their own study ${ }^{3}$ and contact the author of another ${ }^{4}$ to present previously unpublished information. The main thrust of our article is that usual care should be adequately described in any published paper so that the average reader can understand how the study participants and their own patients compare. It should not be necessary to have to make further enquiries.

Given this, I am grateful to Froud and Underwood for the data that they have already supplied to us. This new information adds to our understanding of the course of chronic back pain. While agreeing that their new data show a trend for chronic back pain sufferers in their study to improve over time, I would beg to differ, however, with their conclusion that it 'more closely mimics the acute/sub-acute trajectories'. The information about the UK BEAM study ${ }^{3}$ that they sent to me after the publication of our article gave RMDQ scores for patients with acute, mixed, and chronic duration of back pain. From this I calculate the following falls in RMDQ scores at 3, 6, and 12 months respectively: acute $(1.66,1.3,0.26)$, mixed $(1.45,0.94,0.66)$, and chronic $(1.31,0.7,0.91)$. This would seem to back up our conclusion that 'the longer the duration of the pain, the slower the rate of improvement,' certainly in the first 6 months. The falls in RMDQ score between 6 and 12 months are less than one point which is unlikely to be clinically significant. Data obtained from Licciardone et al by Froud and
Underwood show that RMDQ scores of patients with chronic back pain fell by only by 1.1 in 24 weeks, which the original authors described as showing 'no significant decrease'. ${ }^{4}$ The overall picture remains that the outlook for patients with chronic back pain is less favourable than those with more acute onset.

Despite these very valid points, the overwhelming message that arose from our review remains true. The content and outcome of usual care of low back pain in primary care is not well described and is quite variable. This has implications for research when usual care is employed as a control, but also when considering how to bring evidence-based medicine into routine primary care.

\section{Simon Somerville,}

ARC National Primary Care Centre, Keele University, Keele, Staffordshire, ST5 5BG. E-mail: s.j.somerville@cphc.keele.ac.uk

\section{REFERENCES}

1. Meng CF, Wang D, Ngeow J, et al. Acupuncture for chronic low back pain in older patients: a randomized, controlled trial. Rheumatology (Oxford) 2003; 42(12): 1508-1517.

2. Somerville S, Hay E, Lewis M, et al. Content and outcome of usual primary care for back pain: a systematic review. Br J Gen Pract 2008; 58(556): 790-797, i-vi.

3. UK BEAM Trial Team. United Kingdom back pain exercise and manipulation (UK BEAM) randomised trial: effectiveness of physical treatments for back pain in primary care. BMJ 2004; 329(7479): 1377.

4. Licciardone JC, Stoll ST, Fulda KG, et al. Osteopathic manipulative treatment for chronic low back pain: a randomized controlled trial. Spine 2003; 28(13): 1355-1362.

DOI: 10.3399/bjgp09X419583

\section{Authorship of editorials}

The February 2009 issue of the British Journal of General Practice provides some interesting tensions that reflect the insecurities that Donald Berwick alludes to in the John Hunt lecture 2008. ${ }^{1}$ The RCGP News for February $2009^{2}$ rightly has as its headline that academic general practice is the 'best in the world', that primary care researchers have performed outstandingly. In the journal itself are three articles on depression and primary care, and an editorial on the same topic. The editorial, ${ }^{3}$ written by academic psychiatrists, reviews the three articles, and then provides an opinion about what primary care clinicians should be doing in the consultation: 'Managing depression in primary care: it's not only what you do, it's the way that you do it' is the helpful title.

The question that I ask myself is why do we need to have an editorial from academic psychiatrists, when we know that academic general practice is so strong? We are fortunate to have at least six Professors of General Practice in England, who have built their careers based on research into common mental health conditions, who are more than qualified to have penned the editorial. Is it a feeling, a sense, that general practice needs the specialist view to endorse the findings from primary care? That without this endorsement, and acknowledgement, the findings are in some way diminished? The reverse is true. Academic medicine is not the same as day-to-day clinical care, and psychiatry is not the same as general practice. We do not need the endorsement of psychiatrists to either review the findings, or to give us their opinion on how we should consult. We should be acknowledging that we do manage people with common mental health problems in primary care, very frequently without the benefit of specialist services, and that this care is some of the best in the world.

If we are to understand the meaning and implications of the articles on managing depression in primary care, then asking an academic psychiatrist is as relevant as asking an academic ENT surgeon how to manage children with acute otitis media. In both cases, it is the GP who has the most experience and knowledge of the topic, not the specialist. The ENT surgeon rarely sees children with acute otitis media, and psychiatrists rarely see people with depression and anxiety who are managed in primary care. 